\title{
Catastrophic complication following injection and extracorporeal shock wave therapy of a medial femoral condyle subchondral cystic lesion in a 14 year old Arabian mare
}

\author{
Darla K. Moser ${ }^{1}$, Mike J. Schoonover ${ }^{2, *}$, Kate M. Sippel ${ }^{3}$, Alix M. Dieterly ${ }^{4}$, Jerry W. Ritchey ${ }^{4}$ and Corey R. Wall ${ }^{2}$ \\ ${ }^{1}$ Desert Pines Equine Medical and Surgical Center, Las Vegas, Nevada, USA \\ ${ }^{2}$ Oklahoma State University, Center for Veterinary Health Sciences, Department of Veterinary Clinical Sciences, \\ Stillwater Oklahoma, USA \\ ${ }^{3}$ Gulf Coast Veterinary Specialists, Houston, Texas, USA \\ ${ }^{4}$ Oklahoma State University, Center for Veterinary Health Sciences, Department of Veterinary Pathobiology, \\ Stillwater Oklahoma, USA
}

\begin{abstract}
This report describes fibrous cyst lining injection and extracorporeal shock wave therapy (ESWT) of a medial femoral condyle (MFC) subchondral cystic lesion (SCL) resulting in catastrophic MFC fracture in an Arabian mare. The mare was presented for evaluation of a severe hind limb lameness of approximately 4 months duration. On presentation, a non-weight bearing lameness of the left hind limb with severe effusion and soft tissue swelling of the stifle region was noted. Radiographic evaluation of the stifle revealed a large SCL of the MFC with associated osteoarthritis. Arthroscopic guided intra-lesional injection of the SCL with corticosteroids and autologous bone marrow concentrate was performed followed by ESWT of the MFC. The mare was discharged walking comfortably 48-hours postoperatively. An acute increase in lameness was noted 14 days post-operatively. Imaging revealed catastrophic fracture of the left MFC. Possible mechanisms leading to failure of the MFC secondary to the described treatment are discussed.
\end{abstract}

Keywords: Equine, Extracorporeal shock wave therapy, Intra-lesional injection, Stifle, Subchondral cystic lesion.

\section{Introduction}

Subchondral cystic lesions (SCLs) of the medial femoral condyle (MFC) are a common cause of lameness in horses of all disciplines (Baxter, 1996). They are often diagnosed in horses $<3$ years of age as a manifestation of osteochondrosis but SCLs can also be identified in older horses with trauma as the likely predisposing etiology (Baxter, 1996).

Many different treatment modalities exist ranging from conservative therapies such as stall rest and antiinflammatories to surgical interventions such as cyst debridement or transcondylar screw placement.

Historically, surgical debridement is considered the standard of treatment for horses intended for performance (Mcllwraith, 2013) with success reported as high as 64\% (Sandler et al., 2002). More recently, injection of the fibrous tissue lining of the SCL with corticosteroids has demonstrated success as improvement in lameness in horses diagnosed with SCL in the MFC (Wallis et al., 2008). Advantages of this technique are shorter convalescence, lower risk of cystic enlargement and minimal disruption of the cartilage at the articular surface (Wallis et al., 2008). Extracorporeal shock wave therapy (ESWT) is used clinically to treat a variety of equine conditions including, osteoarthritis, desmitis/tendonitis and stress or non-union fractures (McClure and Weinberger, 2003; Kaneps, 2016).

The introduction of focused shock waves results in compression and sheer loads at tissue interfaces stimulating bone and soft tissue healing by increasing local growth factor production and angiogenesis and reducing inflammation (Kaneps, 2016). Reduction in pain has also been shown experimentally following ESWT (Dahlberg et al., 2006).

Clinical management of MFC SCLs is usually based on the owner's financial constraints, the horse's current or intended use and the size and configuration of the SCL. This report describes catastrophic failure of the MFC following intra-lesional injection, ESWT, and aggressive post-operative pain management of a very large SCL.

\section{Case Details}

A 14-year-old, $458 \mathrm{~kg}$ Arabian mare presented to the Oklahoma State University, Boren Veterinary Medical Teaching Hospital for evaluation of a severe left hind limb lameness. The mare was a retired endurance horse kept in pasture turnout and her intended use was as a broodmare. The owners reported the lameness to have been present for approximately 4 months but was 
exacerbated by a fall 3 months prior to presentation. Conservative therapy consisting of stall rest and intermittent NSAID administration resulted in no improvement over this period prompting veterinary evaluation.

\section{Physical and diagnostic examinations}

On presentation, the mare displayed an American Association of Equine Practitioners (AAEP) grade 5/5 left hind limb lameness. Severe effusion, soft tissue swelling and edema were noted in the stifle area which extended distally toward the tarsus.

No palpable increase in heat was noted but the mare was very painful and resistant to stifle manipulation. Aside from the lameness and inflammation of the stifle and tarsus, no other physical examination abnormalities were noted. The mare displayed a body condition score of 4/9 with a healthy hair coat but a poor appetite.

A radiographic series (lateromedial, caudocranial and caudolateral craniomedial oblique) of both the left and right stifles were obtained using a CPI Millenia Telescoping (3 Phase) radiograph unit with a SoundEklin Mark III digital component. Radiographic findings of the left stifle (Fig. 1) included an approximately $3.1 \mathrm{~cm} \times 2.1 \mathrm{~cm} \times 2.3 \mathrm{~cm}$ in diameter ovoid lucency with surrounding sclerosis noted within the MFC at the articular surface, several subtle smaller rounded lucencies adjacent to the large ovoid lucency measuring approximately $0.4 \mathrm{~cm}$ and $0.3 \mathrm{~cm}$ each, a large smoothly marginated osteophyte present at the proximal medial tibia and significant increase in soft tissue opacity present within the medial femorotibial joint.

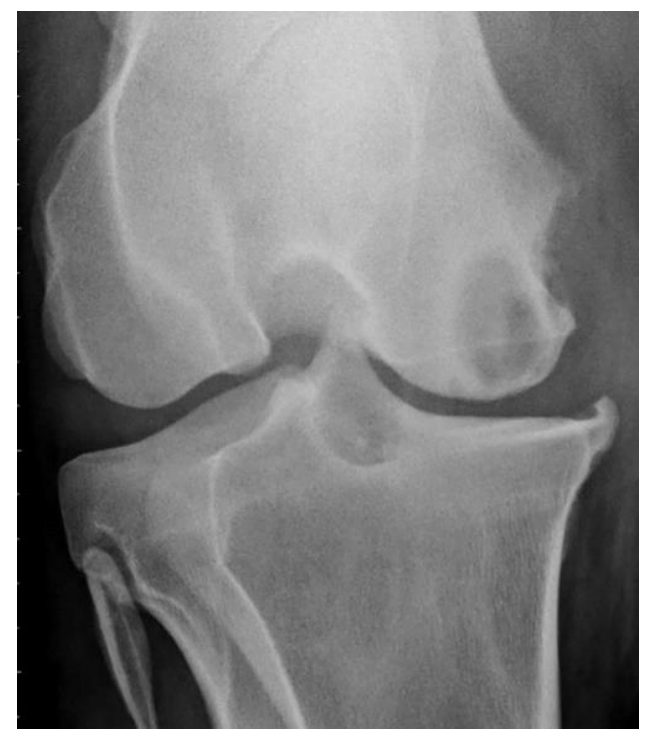

Fig. 1. Caudocranial radiograph performed on initial presentation, revealed a large subchondral bone cyst (SBC) in the left medial femoral condyle (MFC) with secondary osteoarthritis. Enthesiopathy of the distal medial femur and proximal medial tibia.
These findings were consistent with the diagnosis of at least one large grade 4 (Santschi et al., 2015) MFC SCL with secondary effusion and osteoarthritis of the left femorotibial joint. Differentials for the smaller lucencies adjacent to the large SCL included smaller SCLs with secondary sclerosis secondary to joint degeneration. Radiographic findings of the right stifle included a large rounded mineralized body within the medial meniscus and medial collateral ligament, caudomedial periarticular osteophyte formation and increased soft tissue opacity with the medial femorotibial joint. These findings were consistent with the diagnosis of osteoarthritis.

An ultrasound evaluation was performed to better evaluate the soft tissues of the left stifle using a Hitachi Aloka machine with an L64 linear probe (5-18 MHz). A large articular subchondral defect was visualized on the MFC with bony proliferation of the distal femur. Diffuse marked joint effusion, thickened synovium and an abnormal shaped medial meniscus was present. The medial meniscus was bulging and abnormally thin between the MFC and the medial tibia.

\section{Treatment}

Based on the severe degree of lameness, the radiographic and ultrasonographic findings; the owners were given a very poor prognosis for long term improvement in lameness using any currently recommended treatment modalities and counseled that humane euthanasia should be considered. Since the intended use of the mare was for breeding, the owners opted to proceed with treatment consisting of left medial femorotibial (MFT) joint arthroscopic exploration, intra-lesional injection of the fibrous tissue lining of the MFC SCL with corticosteroids and autologous bone marrow concentrate (ABMC) followed by ESWT of the MFC.

Procaine penicillin G $(22,000 \mathrm{IU} / \mathrm{kg} \mathrm{IM})$, gentamicin $(6.6 \mathrm{mg} / \mathrm{kg} \mathrm{IV})$, phenylbutazone $(4.4 \mathrm{mg} / \mathrm{kg} \mathrm{IV)}$ and tetanus prophylaxis was administered pre operatively. Following premedication with xylazine $(0.6 \mathrm{mg} / \mathrm{kg} \mathrm{IV})$ and butorphanol $(0.022 \mathrm{mg} / \mathrm{kg}$ IV) the patient was induced with ketamine $(2.2 \mathrm{mg} / \mathrm{kg} \mathrm{IV})$ and midazolam $(0.11 \mathrm{mg} / \mathrm{kg}$ IV) and maintained with isoflurane delivered in $100 \%$ oxygen. The patient was placed in dorsal recumbency on the surgery table and the left stifle and sternum were aseptically prepared. Bone marrow concentrate was aspirated from the sternum and processed with an Angel System (Arthrex, Naples FL). Simultaneously, arthroscopic evaluation of the left MFT joint was performed through a lateral approach revealing moderate cartilage erosion with fibrin clots within the joints. The SCL in the MFC was identified under arthroscopic visualization and an $18 \mathrm{~g} \mathrm{x} 3$ " spinal needle was inserted into the joint through the articular cartilage into the fibrous tissue periphery of the SCL as described by Wallis et al. (2008). A total of 25mg of 
triamcinolone acetate was injected at 3 different locations followed by injection of $4 \mathrm{mls}$ of ABMC at a separate single location within the fibrous tissue lining of the SCL. Following routine closure of the arthroscopic portal, ESWT (1500 pulses at $0.16 \mathrm{~mJ} / \mathrm{mm}^{2}$ with the NeoVet Shockwave Unit) was performed focusing on the MFC. Recovery from anesthesia was assisted via head and tail ropes and considered uneventful. Antimicrobial therapy was continued for 24 hours post-operatively, phenylbutazone $(4.4 \mathrm{mg} / \mathrm{kg})$ was administered twice daily for 5 days post operatively followed by a reduced dose $(2.2 \mathrm{mg} / \mathrm{kg}$ PO) twice daily for 7 days. Gabapentin $(17 \mathrm{mg} / \mathrm{kg}$ PO) was also prescribed twice daily until reevaluation for added pain management. The patient was discharged from the hospital 48 hours post operatively with instructions for strict stall confinement followed by reevaluation in 14 days. On discharge the mare was fully weight-bearing with no lameness observed at a walk. Ten days following hospital discharge, twelve days post-operatively, the owners noted the mare to be substantially more lame in her stall and with an increase in swelling around her stifle region. Fourteen days post-operatively the mare returned to the hospital for reevaluation. On presentation, she was again non-weight bearing lame (AAEP grade 5/5) on the left hind limb with more extensive swelling noted on the medial aspect of the left stifle. Radiographic (Fig. 2) and ultrasonographic studies of the left stifle were performed. Radiographic findings of the left stifle included complete collapse of the MFT joint and fracture of the medial femoral condyle with wispy bony foci noted within the markedly thickened extracapsular soft tissues of the medial stifle. This was consistent with left medial condylar fracture with joint capsule rupture.

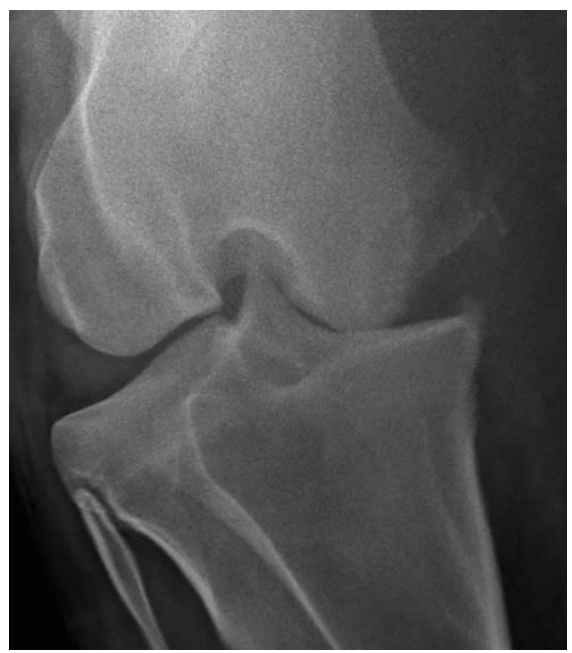

Fig. 2. Caudocranial radiograph of the left stifle performed 14 days post-operatively revealing joint collapse and fracture of the medial aspect of the distal medial femoral condyle.
Ultrasound examination included markedly thickened soft tissues of the left medial aspect of the stifle with numerous distally shadowing mineral foci within thickened soft tissues. The medial meniscus was not visible within the stifle joint. Marked irregularity of the articular surface of the MFC was noted and the medial collateral ligament (MCL) was moderately irregular. Given the degree of damage to the MFT joint and the degree of discomfort displayed by the patient, the owners opted for humane euthanasia.

\section{Post mortem additional diagnostics}

Additional diagnostics including computed tomography (CT), magnetic resonance imaging (MRI) and both gross and histopathology were performed to better evaluate and characterize the stifle lesion. CT of the left stifle was performed using a GE Lightspeed, 4 slice helical CT scanner (Fig. 3a and 3b).
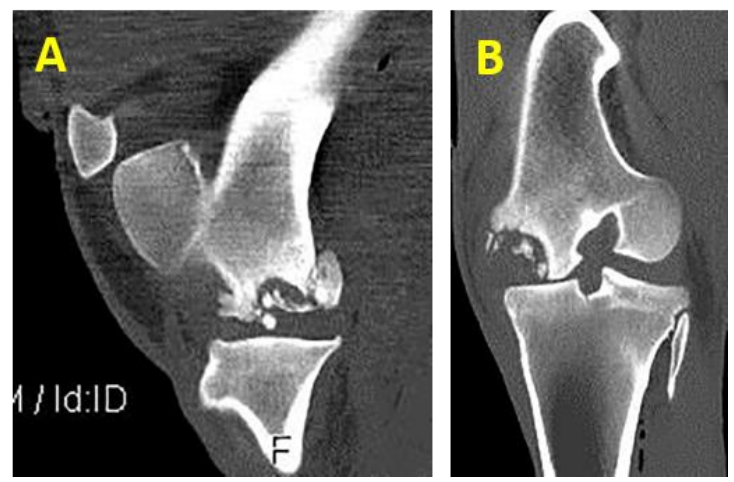

Fig. 3. (A): Sagittal computed tomography image performed post mortem showing fracture of the left medial femoral condyle with multiple pieces of subchondral bone within the medial femorotibial joint capsule. (B): Dorsal computed tomography image performed post mortem showing fracture of the abaxial aspect of the left medial femoral condyle with multiple fragments.

Findings included an approximately $3.2 \mathrm{~cm}$ in diameter rounded lucent region noted within the mid articular margin of the medial condyle. The abaxial aspect of the medial condyle was fractured with numerous bone fragments noted within the surrounding thickened soft tissues as well as a large fragment noted within the large rounded lucency within the medial condyle itself and secondary surrounding sclerosis was noted. An increase of soft tissue opacity was noted within the area of the joint extending medially and caudally containing multiple mineral opacities presumed to be the aforementioned fractures fragments. Bony proliferation along the medial aspect of the proximal tibia was present consistent with osteoarthritis. The MCL of the femorotibial joint could not be definitively identified. This was consistent with the radiographic and ultrasonographic findings. MRI of the left stifle was performed with a GE 1.5 Tesla Echospeed SR 120 MRI with a signa operating format (Fig. 4). 


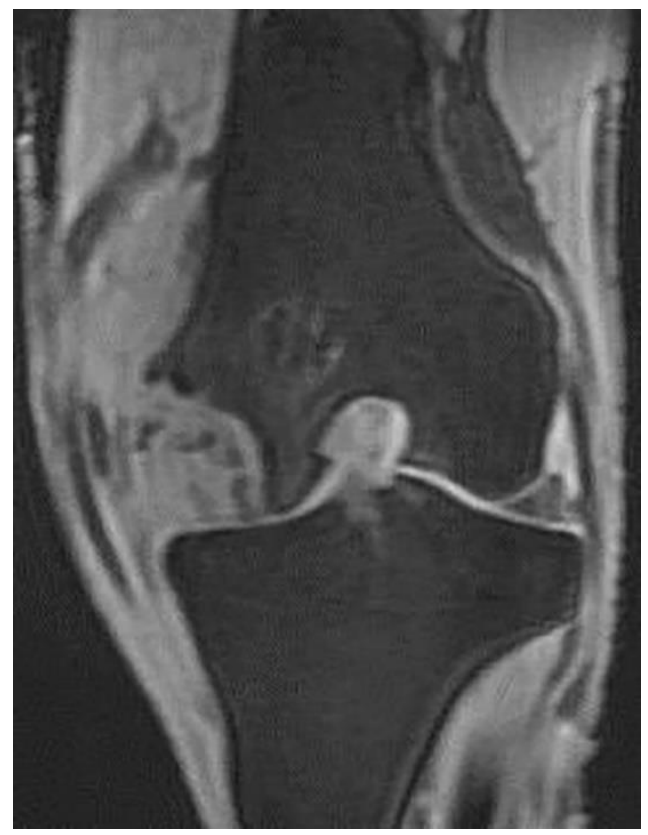

Fig. 4. Dorsal Coronal T1 FAME (fast acquisition with multiphase elliptical fast gradient echo) magnetic resonance image performed post mortem illustrating fracture of the left medial femoral condyle and soft tissue disruption of the medial aspect of the medial femorotibial joint.

The abaxial aspect of the MFC was absent with an irregular margin to the remaining axial aspect. In all sequences, regional hyperintensity was noted throughout the remaining MFC extending proximally into a large portion of the distal femoral metaphysis.

Heterogenous hyperintense soft tissue swelling originating directly from the medial femorotibial joint and extending into the medial, distal and proximal fascia with displacement of the regional musculature was present. The previously described numerous sharply marginated fracture fragments were present within the adjacent soft tissues.

The distal remnants of the MCL were identified; however, the proximal aspect of the MCL was not. Only the most axial and caudal aspects of the medial meniscus was delineated.

MRI findings were consistent with other modalities but provided further information of the left stifle in regards to extensive regional bone edema, loss of abaxial aspect of the medial meniscus and avulsion of the MCL.

\section{Gross pathology:}

A full necropsy examination including detailed evaluation of the left stifle was performed. The MCL and medial joint capsule of the MFT joint were completely ruptured. The articular surface of the MFC of the left hind limb was dull, light tan and finely granular. The caudolateral region of the condyle was fragmented into multiple variously shaped and sized pieces causing a depression in the condylar silhouette (Fig. 5a).
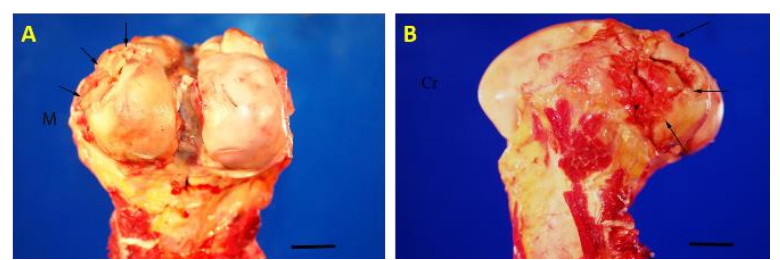

Fig. 5. (A): Caudal view of the femur with a 2 centimeter scale bar in the lower right corner. The medial femoral condyle is diffusely dull, tan/yellow with a granular articular surface and the articular cartilage of the craniomedial region (arrowheads) is fragmented and collapsed. $M$ indicates medial. (B): Medial view of the femur with a 2 centimeter scale bar in the lower right corner. The articular cartilage of the craniomedial region aspect of the medial femoral condyle (arrowheads) is fragmented and collapsed. $\mathrm{Cr}$ indicates cranial.

Upon cross section, an approximately $3 \mathrm{~cm} \times 2 \mathrm{~cm}$ round cavitary lesion was present in the subchondral bone in this region (Fig. 5b and 6). The lesion consisted primarily of gray, firm tissue replacing the subchondral bone and the articular cartilage overlying the subchondral lesion was thinned. The majority of the cranial medial meniscus was nonexistent and the remaining caudal portion was macerated, frayed and hemorrhagic. The periarticular connective tissue contained abundant hemorrhage and fibrin. A hemorrhagic dissecting tract was present extending through muscle fascia from the MFC to approximately mid femur.

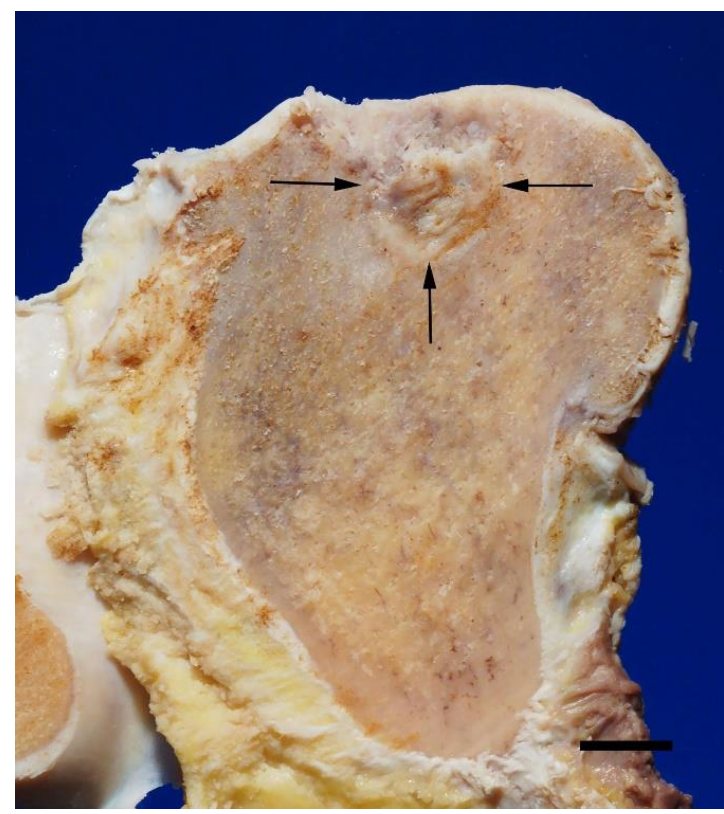

Fig. 6. Medial femoral condyle, sagittal section after formalin fixation with a 2 centimeter scale bar in the lower right corner. The sagittal section through the medial femoral condyle reveals a subchondral bone cyst comprised primarily of fibrous connective tissue replacing the subchondral bone (arrowheads). 


\section{Histopathology:}

Histopathology of the MFC lesion revealed replacement of the normal subchondral bone with fibrous connective tissue and organized, mature granulation tissue. Peripherally, the fibrous connective tissue and granulation tissue blended into fibrocartilage at the junction to the neighboring bone.

\section{Discussion}

The size of the SCL as well as the age and intended use of the patient in this case prompted the authors to choose a more conservative approach rather than extensive surgical debridement or placement of a transcondylar screw. The SCL occupied approximately $30 \%$ of the MFC which was concerning from a biomechanical loading view point. Equine stifle joint loading properties have not yet been defined but it is reasonable to infer that a large SCL such as the one in the present case would have a significant influence on the normal MFC biomechanics. It was our opinion that debridement of the SCL would further weaken the medial condyle possibly resulting in a catastrophic fracture. Unfortunately, this was not prevented even with what was assumed to be a more conservative approach.

To the authors' knowledge, this is the first report of a fracture of the medial femoral condyle following treatment of a SCL with intra-lesional corticosteroid injection. In addition to the SCL injection, ESWT was performed and systemic analgesic medications were administered to this patient. Due to the severity of the lameness on presentation and concern for contralateral limb complications, an aggressive analgesic protocol was employed pre and post operatively.

Upon dismissal and throughout the post-operative period the mare was observed to be significantly more comfortable until she was found non-weight bearing on day 12 . The analgesic properties of phenylbutazone are well defined (Foreman et al., 2008) and gabapentin was administered to address any neuropathic pain stimulated by the chronicity of the lameness. The oral bioavailability of gabapentin is relatively low and oral doses as high as $20 \mathrm{mg} / \mathrm{kg}$ have resulted in no apparent adverse side effects in horses (Terry et al., 2010) but the analgesic properties of gabapentin are yet to be clearly established in horses. It is possible that the postoperative analgesic protocol used in this case could have increased the patient's comfort on the affected limb to the point of over-exertion resulting catastrophic failure of the MFC.

The analgesic properties of ESWT on bone pain have been demonstrated in several experimental studies (Dahlberg et al., 2006; Kawcak et al., 2011). The exact time of onset and duration of analgesia resulting from ESWT has not been firmly established in the horse, but many racing jurisdictions and the International Federation of Equestrian Sport (FEI) regulations prohibit the use of ESWT within 5 days of a competition (USEF, 2016). Although no scientific reports describe the use of ESWT for the treatment of MFC SCLs, one of the authors (MJS) has used this modality in combination with cyst injection in a number of horses without complication. It should be noted however that the size of the SCL in this report was much larger than others treated with injection and ESWT.

It is feasible that the post ESWT analgesia achieved in the immediate post-operative period increased the mare's weight bearing on the limb thus loading the already weakened MFC to the point of failure. Additionally, significant microfractures could have been induced in the already compromised MFC by the ESWT leading to further mechanical weakening of the structure. The perceived risk of catastrophic fracture of the MFC with surgical debridement was discussed with the owner pre-operatively and many treatment options as well as euthanasia were discussed. Catastrophic fracture of the MFC unfortunately occurred following the treatments described here. The size of the SCL in this case was very large and we believe this played a major role in the ultimate outcome.

It is our opinion that the post-operative analgesia achieved with the combination of arthroscopic lavage, injection of the SCL lining, ESWT and systemic analgesics promoted increased weight bearing and this increased weight bearing led to catastrophic fracture of the MFC. The severe lameness displayed by this mare on presentation required aggressive pain management along with treatment of the SCL for humane reasons as well as to decrease the chance of contralateral limb complications.

We cannot rule out the possibility of a non-related traumatic accident in the stall leading to fracture independent of all treatment therapies. In very large MFC SCLs similar to the one presented here, treatment modalities such as intra-lesional injection of the fibrous cyst lining and analgesic protocols used following treatment should be carefully evaluated to provide appropriate analgesia but not encourage immediate full loading of an already weakened MFC.

\section{Conflict of interest:}

The authors declare that there is no conflict of interest.

\section{References}

Baxter, G.M. 1996. Subchondral cystic lesions in horses. In Joint disease in the horse, Eds., McIlwraith, CW. and Trotter, GW. Philadelphia: WB Saunders, pp: 384-397.

Dahlberg, J.A., McClure, S.R., Evans, R.B. and Reinertson, E.L. 2006. Force plate evaluation of lameness severity following extracorporeal shock wave therapy in horses with unilateral forelimb lameness. J. Am. Vet. Med. Assoc. 229, 100-103. 
Foreman, J.H., Barange, A., Lawrence, L.M. and Hungerford, L.L. 2008. Effects of single-dose intravenous phenylbutazone on experimentally induced, reversible lameness in the horse. J. Vet. Pharmacol. Therap. 31, 39-44.

Kaneps, A.J. 2016. Practical rehabilitation and physical therapy for the general equine practitioner. Vet. Clin. North. Am. Equine Pract. 32, 167-180.

Kawcak, C.E., Frisbie, D.D. and McIlwraith, C.W. 2011. Effects of extracorporeal shock wave therapy and polysulfated glycosaminoglycan treatment on subchondral bone, serum biomarkers, and synovial fluid biomarkers in horses with induced osteoarthritis. Am. J. Vet. Res. 72, 772-779.

McClure, S.R. and Weinberger, T. 2003. Extracorporeal shock wave therapy: Clinical applications and regulation. Clin. Tech. Equine Pract. 2, 358-367.

McIlwraith, C.W. 2013. Surgical versus conservative management of osteochondrosis. Vet. J. 197, 19-28.

Sandler, E.A., Bramlage, L.R., Embertson, R.M., Ruggles, A.J. and Frisbie, D.D. 2002. Correlation of lesion size with racing performance in thoroughbreds after arthroscopic surgical treatment of subchondral cystic lesions of the medial femoral condyle: 150 cases. In the proceedings of the 2002
Annual Convention of the American Association of Equine Practitioners, pp: 255-256.

Santschi, E.M., Williams, J.M., Morgan, J.W., Johnson, C.R., Bertone, A.L. and Juzwiak, J.S. 2015. Preliminary investigation of the treatment of equine medial femoral condylar subchondral cystic lesions with a transcondylar screw. Vet. Surg. 44, 281-288.

Terry, R.L., McDonnell, S.M., VanEps, A.W., Soma, L.R., Liu, Y., Uboh, C.E., Moate, P.J. and Driessen, B. 2010. Pharacokinetic profile and behavioral effects of gabapentin in the horse. J. Vet. Pharmacol. Therap. 33, 485-494.

United States Equestrian Federation (USEF). 2016. 2017 Guidelines for drugs and medications. pg. 3. United States Equestrian Federation Equine Drugs and Medications Program, Columbus, $\mathrm{OH}$. https://www.usef.org/documents/drugsMeds/2017/ 2017Guidelines.pdf.

Wallis, T.W., Goodrich, L.R., McIlwraith, C.W., Frisbie, D.D., Hendrickson, D.A., Trotter, G.W., Baxter, G.M. and Kawcak, C.E. 2008. Arthroscopic injection of corticosteroids into the fibrous tissue of subchondral cystic lesions of the medial femoral condyle in horses: A retrospective study of 52 cases (2001-2006). Equine Vet. J. 40, 461-467. 